OPEN ACCESS

Edited by:

Huijie Bian,

Fourth Military Medical University,

China

Reviewed by:

Lihua Chen,

Fourth Military Medical University,

China

Yan Wu,

Sun Yat-sen University, China

${ }^{*}$ Correspondence:

Weishan Chen

chenweishan@zju.edu.cn

Ning Zhang

zhangning@zju.edu.cn

tThese authors have contributed equally to this work

Specialty section:

This article was submitted to

Cell Death and Survival,

a section of the journal

Frontiers in Cell and Developmental

Biology

Received: 19 May 2020 Accepted: 11 August 2020

Published: 31 August 2020

Citation:

Xu Q, Xing $H, W u J$, Chen W and Zhang N (2020) miRNA-141 Induced Pyroptosis in Intervertebral Disk

Degeneration by Targeting ROS

Generation and Activating TXNIP/NLRP3 Signaling in Nucleus

Pulpous Cells.

Front. Cell Dev. Biol. 8:871. doi: 10.3389/fcell.2020.00871

\section{miRNA-141 Induced Pyroptosis in Intervertebral Disk Degeneration by Targeting ROS Generation and Activating TXNIP/NLRP3 Signaling in Nucleus Pulpous Cells}

\author{
Qiaolong $\mathrm{Xu}^{1,2 t}$, Hongyuan Xing ${ }^{1 \dagger}$, Jiaqi Wu ${ }^{1}$, Weishan Chen ${ }^{1 *}$ and Ning Zhang ${ }^{1 *}$ \\ 1 Department of Orthopedics, 2nd Affiliated Hospital, School of Medicine, Zhejiang University, Hangzhou, China, \\ 2 Department of Orthopaedics, The People's Hospital of Cixi, Cixi, China
}

The role and mechanism of pyroptosis in intervertebral disk (IVD) degeneration are unclear. MicroRNAs (miRNAs) regulate the viability and function of nucleus pulposus cells (NPCs) in IVDs and are related to pyroptosis. We performed microarray analyses of normal and degenerated nucleus pulposus (NP) to assess the role of pyroptosis and identify key miRNAs in IVD degeneration. We also evaluated the underlying mechanism of miRNA-mediated pyroptosis in NPCs. In addition, we demonstrated the preventative effects of miRNAs on IVD degeneration in a rat model. The levels of the pyroptosis-related proteins cleaved caspase-1, N-terminal gasdermin D (GSDMD), interleukin (IL)-1 $\beta$, and IL-18 in the degenerative NP were significantly higher than those in the normal NP. miRNA-141 was significantly upregulated in the degenerated NP. miR-141 mimic suppressed the matrix synthesis function of NPCs. By contrast, reactive oxygen species (ROS) generation, and the expression of TXNIP and NLRP3 were significantly downregulated by an miR-141 inhibitor. Furthermore, the miRNA141 inhibitor prevented the degeneration of IVDs in vivo. Our findings suggest that miRNA-141 induces pyroptosis and extracellular matrix (ECM) catabolism in NPCs by increasing ROS generation and activating TXNIP/NLRP3 signaling. miRNA-141regulated pyroptosis may be a novel therapeutic target for IVD degeneration.

Keywords: extracellular matrix, intervertebral disk degeneration, miRNA-141, pyroptosis, TXNIP/NLRP3 signaling

\section{INTRODUCTION}

Intervertebral disk (IVD) degeneration and the consequent chronic low-back pain affect approximately 632 million people worldwide and exert social and economic effects on patients (Mathew et al., 2013). IVD degeneration imposes the highest economic burden among all musculoskeletal complaints (Manchikanti et al., 2009). The IVD, which is composed of the nucleus pulposus (NP), annulus fibrosus (AF), and cartilaginous endplates, contributes to motion, weight 
bearing, and flexibility while protecting the spinal cord (Bowles and Setton, 2017). The healthy NP contains normal NP cells (NPCs), which synthesize collagen II and proteoglycan. The dysfunction of NPCs and alteration of the extracellular matrix (ECM) is thought to be the origin of IVD degeneration (Kepler et al., 2013).

Many factors regulate the viability and function of NPCs. Apoptosis is one of the main causes of NPC loss and dysfunction (Song Y. et al., 2018). Apoptosis of NPCs leads to downregulation of ECM synthesis by activating the p53 signaling pathway (Jin et al., 2018). Apoptosis is also involved in several grades of IVD degeneration (Eser et al., 2017). Other factors, such as nutrients and aging, may also regulate the viability of, and ECM synthesis by NPCs (Barakat et al., 2019). In recent years, a number of studies have focused on pyroptosis, a novel inflammatory form of regulated cell death (Liu X. et al., 2016; Ruhl et al., 2018).

Canonical pyroptosis is typically mediated by active caspase-1, which cleaves the pore-forming protein gasdermin D (GSDMD). The N-terminal domain is liberated from GSDMD after cleavage and triggers pyroptosis and the release of biologically activate interleukin (IL)-1 $\beta$ and IL-18, generating an inflammasomeassociated inflammatory response (Xue et al., 2019). Pyroptosis occurs in various types of cells and tissues. Zheng et al. (2020) reported a role for GSDME-mediated pyroptosis in cardiac injury. NLRP3-related pyroptosis is involved in the occurrence and development of diabetes mellitus and its associated complications ( $\mathrm{Yu}$ et al., 2020). Endoplasmic reticulum-related pyroptosis plays a role in the pathogenesis of steatohepatitis. In chondrocytes, pyroptosis modulates cell viability and ECM synthesis (Hu et al., 2020). However, the role of pyroptosis in IVD degeneration is unclear. Whether pyroptosis regulates the viability of, and ECM synthesis by, NPCs needs to be investigated.

MicroRNAs (miRNAs) are small endogenous non-coding RNAs of 21-23 nucleotides that regulate gene expression (Bartel, 2009). miRNAs play critical roles in cell proliferation, function, and pyroptosis (Kong et al., 2019; Zeng et al., 2019). In IVDs, miRNAs regulate the viability and function of NPCs. miR532 induces NPC apoptosis by downregulating Wnt/ $\beta$ catenin signaling (Sun et al., 2018). miR-194 the inhibits inflammatory response in NPCs by targeting TNF receptor-associated factor 6 (Kong et al., 2018). miR-7 modulates ECM degeneration of NPCs by targeting growth differentiation factor 5 (Liu W. et al., 2016). We performed microarray analyses of normal and degenerated NPs and found 108 significantly differentially expressed miRNAs $(p<0.01)$. Among them, the level of miR-141 in degenerated NP was significantly higher than that in normal NP. A prior study reported that miR-141 is related to IVD degeneration (Ji et al., 2018). Therefore, miR-141 may be a key regulator in NPCs. miR-141 modulates cell migration, proliferation, and apoptosis (Zhao et al., 2013; Li et al., 2017; Qin et al., 2019). However, whether miR-141 mediates pyroptosis in NPCs and the underlying mechanism are unclear.

We investigated the role of $\mathrm{miR}-141$ in regulating pyroptosis in NPCs during IVD degeneration. We further evaluated the mechanism underlying miR-141-mediated pyroptosis in NPCs. Finally, we assessed the preventative effect of an miR-141 inhibitor on degenerated IVD in a rat model. Discovery of the effect of miR-141 on pyroptosis in NPCs may provide a novel therapeutic target for IVD degeneration.

\section{MATERIALS AND METHODS}

\section{Tissue Source and Cell Culture}

The study was approved by the Ethics Committee of The Second Affiliated Hospital of Zhejiang University School of Medicine, and informed consent was obtained from all participating patients. Normal NPs were donated by 10 patients (10-20 years old) without a clinical history of IVD disease and degenerative NPs by 10 patients (40-50 years old) with clinical characteristics of low back pain and lower limb pain. The disk degeneration grades of the donors were evaluated according to the Pfirrmann classification by magnetic resonance imaging of the IVD. NP samples were obtained by nucleotomy and intervertebral fusion surgery under sterile conditions and processed within $1 \mathrm{~h}$ of being harvested.

Healthy human NPCs were purchased from Procell (Procell Life Science \& Technology, Hubei, China) and cultured in Dulbecco's modified Eagle's medium (DMEM)-high glucose supplemented with $10 \%$ fetal bovine serum (FBS), $4 \mathrm{mM}$ L-glutamine, and $1 \%$ penicillin-streptomycin at $37^{\circ} \mathrm{C}$ in $5 \%$ $\mathrm{CO}_{2}$. NPCs at passages 2-4 were harvested. We used a $\mathrm{pH} 6.8$ solution adjusted with sterilized $\mathrm{HCl}(1 \mathrm{M})$ to simulate the mildly degenerated IVD and induce the degeneration of NPCs (Fu et al., 2018; Hartman et al., 2018).

\section{Cell Transfection}

The human mimic-141 negative control (NC), miR-141 mimic, miR-141 inhibitor NC, and miR-141 inhibitor were purchased from GenePharma (Shanghai, China) and transfected according to the manufacturer's instructions. Briefly, cells were cultured in serum-free DMEM-high glucose medium for $12 \mathrm{~h}$. The miR-NC, miR-mimic, or miR-inhibitor, and Lipofectamine $2000^{\mathrm{TM}}$ transfection reagent were diluted in $250 \mu \mathrm{L}$ of Opti-MEM (Gibco, Shanghai, China). The LipofectaminemiRNA mixture was added to the serum-free medium. Fresh medium containing $10 \%$ FBS was added to stop the transfection after $6 \mathrm{~h}$.

\section{Cell Viability}

The viability of NPCs was assessed using a Cell Counting Kit8 (CCK8, Dojindo, Dalian, China). NPCs were seeded into a 96-well plate. At predetermined time points, the medium was removed, and the cells were treated with $10 \%$ CCK8 in $100 \mu \mathrm{L}$ of DMEM-high glucose for $2 \mathrm{~h}$ at $37^{\circ} \mathrm{C}$. Absorbance at $450 \mathrm{~nm}$ was measured using a microplate reader (Bio-Rad Laboratories, Hercules, CA, United States).

\section{RNA Isolation and Real-Time Quantitative PCR (RT-qPCR)}

Total RNA was extracted from tissues or NPCs using RNAiso reagent (Takara, Shanghai, China) and reverse-transcribed to complementary DNA using PrimeScript ${ }^{\mathrm{TM}}$ RT Master Mix 
TABLE 1 | Primers used in Real-time-quantitative polymerase chain reaction (RT-PCR).

\begin{tabular}{|c|c|c|}
\hline Gene & Forward Primer $\left(5^{\prime}-3^{\prime}\right)$ & Reverse Primer $\left(5^{\prime}-3^{\prime}\right)$ \\
\hline $18 s$ & $\begin{array}{l}\text { GAATTCCCAGTAAGTGC } \\
\text { GGGTCATA }\end{array}$ & $\begin{array}{l}\text { CGAGGGCCTCACTAAAC } \\
\text { CATC }\end{array}$ \\
\hline U6 & CTCGCTTCGGCAGCACA & $\begin{array}{l}\text { AACGCTTCACGAATTTG } \\
\text { CGT }\end{array}$ \\
\hline hsa-miR-141-3p & $\begin{array}{l}\text { GCGGCGGTAACACTGT } \\
\text { CTGG }\end{array}$ & $\begin{array}{l}\text { AACGCTTCACGAATाTG } \\
\text { CGT }\end{array}$ \\
\hline mo-miR-141-3p & $\begin{array}{l}\text { GTAGAAGGTCACGTCA } \\
\text { CAAC }\end{array}$ & CCTAACACTGTCTGGTAA \\
\hline Acan & $\begin{array}{l}\text { CTAGCTGCTTAGCAGGG } \\
\text { ATAACG }\end{array}$ & $\begin{array}{l}\text { GATGACCCGCAGAGTCAC } \\
\text { AAAG }\end{array}$ \\
\hline Sox9 & $\begin{array}{l}\text { AGGAAGCTGGCAGACC } \\
\text { AGTACC }\end{array}$ & $\begin{array}{l}\text { GGGTCTCTTCTCGCTCTC } \\
\text { GTTCA }\end{array}$ \\
\hline Col2a1 & $\begin{array}{l}\text { CTGGTGGAGCAGCAA } \\
\text { GAGC }\end{array}$ & $\begin{array}{l}\text { GTGGACAGTAGACGGAG } \\
\text { GAAAG }\end{array}$ \\
\hline ADAMT4 & $\begin{array}{l}\text { CTCCTGCCTITAGCC } \\
\text { TGGTC }\end{array}$ & $\begin{array}{l}\text { CCCAAAGGCTGGTAATC } \\
\text { GGT }\end{array}$ \\
\hline MMP3 & $\begin{array}{l}\text { TGATGGGCCTGGAAT } \\
\text { GGTC }\end{array}$ & $\begin{array}{l}\text { TTCATGAGCAGCAACCAG } \\
\text { GAATAG }\end{array}$ \\
\hline MMP13 & $\begin{array}{l}\text { TGATGATGAAACCTGG } \\
\text { ACAAGCA }\end{array}$ & $\begin{array}{l}\text { GAACGTCATCATCTGGG } \\
\text { AGCA }\end{array}$ \\
\hline
\end{tabular}

or miRNA First-Strand Synthesis kits. Real-time-quantitative polymerase chain reaction (RT-qPCR) was performed using TB Green Premix Ex Taq (Takara) on an ABI StepOnePlus System (Applied Biosystems, Warrington, United Kingdom). miR-141 expression was normalized to U6, and those of other mRNAs to that of $18 \mathrm{~S}$. The data were analyzed by the $2^{(-\Delta \Delta \mathrm{CT})}$ method and the primers used were synthesized by Sangon Biotech (Shanghai, China; Table 1).

\section{Western Blotting}

Protein was extracted from tissue and cell samples in radioimmunoprecipitation assay buffer supplemented with a proteasome inhibitor (Beyotime, China), and was separated by sodium dodecyl sulfate-polyacrylamide gel electrophoresis. Next, the proteins were transferred to a polyvinylidene fluoride membrane (Millipore, Shanghai, China) by electroblotting. The membranes were blocked with $5 \%$ non-fat milk for $2 \mathrm{~h}$ and incubated overnight with antibodies against cleaved caspase-1 (ab207802, Abcam, Shanghai, China), NT-GSDMD (ab215203, Abcam), IL-1 $\beta$ (12703, Cell Signaling Technology, Shanghai, China), IL-18 (54943, Abcam), TXNIP (ab188865, Abcam), or NLRP3 (ab263899, Abcam). GAPDH (ab181602, Abcam) was used as the internal control. After washing three times with Tris-buffered saline containing $0.1 \%$ Tween-20 (TBST), the membranes were incubated with the horseradish peroxidaseconjugated secondary antibodies (Beyotime) for $1 \mathrm{~h}$ at room temperature. Immunoreactive bands were visualized using an enhanced chemiluminescence substrate (Millipore). Signal intensity was measured using the Bio-Rad XRS chemiluminescence detection system (Bio-Rad laboratories, Hercules, CA, United States).

\section{Microarray Analysis}

Total RNA was extracted from tissues using TRIzol reagent (Takara) and quantified using a NanoDrop ND-2000 (Thermo Fisher Scientific, Shanghai, China). After purified with a QIAGEN RNeasy Kit (QIAGEN, Shanghai, China), total RNA was amplified and labeled with Cy-3. Next, RNA was hybridized for $17 \mathrm{~h}$ at $65^{\circ} \mathrm{C}$ and washed with Gene Expression Wash Buffers 1 and 2 (Agilent Technologies, Beijing, China). Array images were acquired using Agilent Scanner G5761A (Agilent Technologies) and analyzed using Agilent Feature Extraction software (version 12.0.1.1). Quantile normalization and subsequent data processing were performed by using the GeneSpring v14.8 software package (Agilent Technologies). Next, miRNAs with at least three out of the six samples having flags in Detected were chosen for further analysis. Significantly differentially expressed miRNAs $(p<0.01)$ were identified and subjected to hierarchical cluster analysis. Heat map and gene ontology (GO) enrichment analyses were performed to identify the biological functions of the differentially expressed miRNAs.

\section{Immunofluorescence Staining}

Nucleus pulposus cells were cultured in 12-well plates. After fixation with $4 \%$ paraformaldehyde for $10 \mathrm{~min}$ at room temperature, the cells were treated with $0.1 \%$ Triton X-100 for $10 \mathrm{~min}$ and incubated with $2 \%$ bovine serum albumin for $30 \mathrm{~min}$ at room temperature. Next, cells were incubated with anti-collagen II (ab34712, Abcam) and anti-aggrecan antibodies (ab3773, Abcam) overnight and then incubated with an Alexa Fluor 555-labeled secondary antibody for $1 \mathrm{~h}$ at room temperature in the dark. Nuclei were stained with 4',6-diamidino-2-phenylindole (DAPI, Sigma-Aldrich, Shanghai, China) for $5 \mathrm{~min}$. Fluorescence was detected using a fluorescence microscope (DM5500; Leica, Wetzlar, Germany).

\section{Alcian Blue Staining}

Nucleus pulposus cells were cultured in six-well plates for 7 days. The cells were fixed with $4 \%$ paraformaldehyde for $10 \mathrm{~min}$ and incubated in Alcian blue staining solution (Sigma-Aldrich) for $30 \mathrm{~min}$ at room temperature. After washing three times with distilled water, three fields per well were chosen randomly for observation under an inverted microscope (Leica).

\section{Detection of Cellular Reactive Oxygen Species (ROS)}

Nucleus pulposus cells were cultured in 12-well plates. After treatment with acid or N-acetyl cysteine (NAC, Sigma-Aldrich), the NPCs were rinsed and incubated with $5 \mu \mathrm{M}$ DCFH-DA (Sigma-Aldrich) in the dark at $37^{\circ} \mathrm{C}$ for $30 \mathrm{~min}$. Fluorescence was detected using a fluorescence microscope (DM5500; Leica).

\section{Flow Cytometry}

Flow cytometry was performed to assess pyroptotic cell death. Fluorochrome inhibitor of caspase-1 (caspase-1 FLICA) and propidium iodide (PI) were added to NPCs, and pyroptotic death was assessed by flow cytometry after FLICA/PI staining. PI $(+)$ and caspase-1 FLICA $(+)$ was defined as pyroptosis. 


\section{Animal and Surgical Procedure}

Male Sprague Dawley rats weighing $250 \mathrm{~g}$ were obtained from the Animal Center of the Academy of Medical Science of Zhejiang Province. All procedures in this study were approved by the Institutional Animal Care and Use Committee of Zhejiang University. Rats were anesthetized with $1 \%$ sodium pentobarbital (Sigma-Aldrich) and a $20 \mathrm{G}$ sterile needle was inserted into the disk of coccygeal vertebrae (Co)7/Co 8 and $\mathrm{Co} 8 / \mathrm{Co} 9$ to a depth of approximately $5 \mathrm{~mm}$. Next, the needle was rotated $360^{\circ}$ and maintained for $30 \mathrm{~s}$ before being removed (Masuda et al., 2005). For NP generation and injection, $3 \mu \mathrm{g}$ of miR-141 mimic or inhibitor was packed in a NP carrier system (MaxSuppressor in vivo RNALANCEr II Kit and Lipid Extruder, BIOO Scientific, Beijing, China). Two weeks after the surgical procedure, 32 rats were randomized into four groups: the normal group (without needle puncture and other treatments); the NC group (with needle puncture and phosphate buffer solution injection); the miR-141 mimic group (with needle puncture and miR-141 mimic injection); and the miR-141 inhibitor group (with needle puncture and miR-141 inhibitor injection). The injection was performed using a microsyringe with a 31-G needle and $3 \mu \mathrm{L}$ of liquid. miR-141 mimic or inhibitor was injected every week.

\section{Histological, Immunohistochemical, and Biochemical Analysis}

Samples of rat tails were harvested at 16 weeks after injection. The samples were fixed in $4 \%$ paraformaldehyde for 3 days before being immersed in decalcifying solution. The samples were dehydrated, embedded in paraffin, and sectioned at $3.5 \mu \mathrm{m}$ thickness using a microtome. For histological analysis, haematoxylin and eosin $(\mathrm{H} \& \mathrm{E})$ and safranine $\mathrm{O}$-fast green staining were performed separately on consecutive tissue sections. Cellularity and morphology were assessed using a previously described grading scale and presented as a histological score (Han et al., 2008). For immunohistochemical analysis, tissue sections were treated with $3 \% \mathrm{H}_{2} \mathrm{O}_{2}$ for $10 \mathrm{~min}$ and blocked with goat serum for $30 \mathrm{~min}$ at room temperature. The tissue sections were incubated with anti-collagen II antibody (Abcam) overnight at $4^{\circ} \mathrm{C}$, then with a biotin-conjugated secondary antibody for $1 \mathrm{~h}$ at room temperature and detected by the SABC method. Images were obtained using a microscope (Leica). For biochemical analysis, NP samples of rats were frozen at $-80^{\circ} \mathrm{C}$ and lyophilized for $24 \mathrm{~h}$, and the dry weight was recorded. The glycosaminoglycan (GAG) content was determined by Blyscan assay (Biocolor, Beijing, China) according to the manufacturer's protocols and normalized to the dry weight.

\section{Statistical Analysis}

All experiments were repeated three times and data are presented as the means \pm standard deviation. A two-tailed Student's $t$-test and one-way analysis of variance followed by Tukey post hoc test were performed to assess the significance of differences using SPSS ver. 22.0 (IBM Corp., Armonk, NY, United States). A value of $p<0.05$ was considered to indicate statistical significance.

\section{RESTULTS}

\section{Pyroptosis Was Related With Disk Degeneration}

We assessed pyroptosis in normal and degenerative NP by western blotting. The protein levels of cleaved caspase-1, NT-GSDMD, IL-1 $\beta$, and IL-18 in the degenerative NP were significantly higher (6.32-, 5. 56-, 9. 52-, and 6.86-fold) than those in the normal NP (Figures 1A,B).

\section{miR-141 Was Highly Expressed in Degenerated NP}

We performed miRNA microarray analysis to identify differentially expressed miRNAs between normal and degenerative NP. Only miRNAs with a mean fold change $>5$ or $<0.2$ and a $p$-value of $<0.01$ were selected for further analysis. Thirty-nine miRNAs were significantly dysregulated; of them, miR-141 was the most significantly upregulated miRNA in the degenerative group compared to the normal group (Figure 2A). Dysregulated mRNAs were also subjected to GO analysis. The results showed that the GO terms with the most significant $p$ values for biological processes, molecular function, and cellular component were related to ECM disassembly (GO:0022617), ECM organization (GO:0030198), platelet degranulation (GO:0002576), and positive regulation of NLRP3 inflammasome complex assembly (GO:1900227) which was involved in the process of pyroptosis (Figure 2B). RT-qPCR showed that the expression level of miR-141 in the degeneration group was almost 20.05-fold that in the normal group (Figure 2C).

\section{miR-141 Induced Pyroptosis in NPCs}

An acidic environment significantly increased the expression of miR-141 (Figure 3A) and the protein levels of cleaved caspase1 , NT-GSDMD, IL-1 $\beta$, and IL-18 (Figure 3B). The expression level of miR-141 was quantified after the transfection of mimicNC, inhibitor-NC, miR-141 mimic, and miR-141 inhibitor. No significant difference was observed between the control mimic-NC, and inhibitor-NC groups. The expression levels of miR-141 in the miR-141 mimic and miR-141 inhibitor groups were 203.71- and 0.14-fold that in the control group (Figure 3C). The viability of NPCs in the miR-mimic group was lower than that in the mimic-NC group at each time point. The miRinhibitor group showed higher cell viability compared to the inhibitor-NC group in an acidic environment, particularly at $72 \mathrm{~h}$ (Figure 3D). We also assessed pyroptosis in NPCs by measuring the protein levels of cleaved caspase-1, NT-GSDMD, IL-1 $\beta$, and IL-18. The miR-141 mimic significantly promoted pyroptosis by increasing the levels of cleaved caspase-1, NT-GSDMD, IL-1 $\beta$, and IL-18; the miR-141 inhibitor prevented pyroptosis by downregulating cleaved caspase-1, NT-GSDMD, IL-1 $\beta$, and IL-18 compared with the inhibitor-NC group (Figures 3E,F).

\section{miR-141 Suppressed the Matrix Synthesis Function of NPCs}

The effect of miR-141 on matrix synthesis by NPCs was evaluated. The synthesis of collagen II and aggrecan was downregulated by 
A

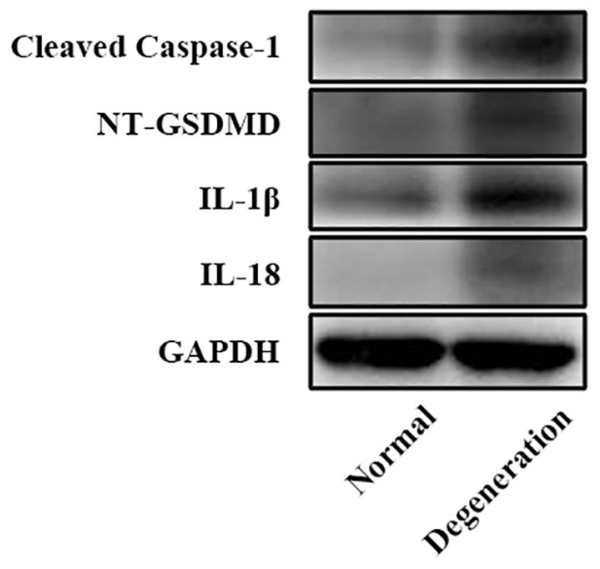

B

$20 \mathrm{kDa}$

$53 \mathrm{kDa}$

$31 \mathrm{kDa}$

$22 \mathrm{kDa}$

$36 \mathrm{kDa}$

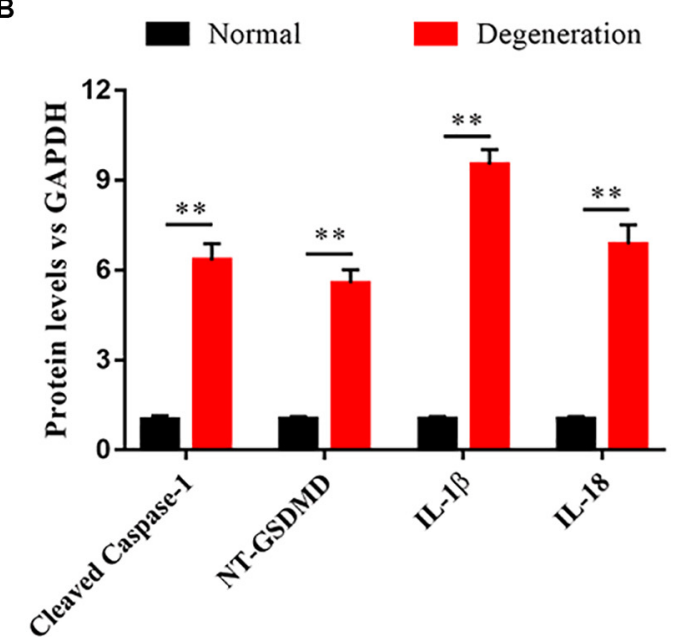

FIGURE 1 | Pyroptosis was related with disk degeneration. (A) Protein expression levels of Cleaved caspase-1, NT-GSDMD, IL-1 $\beta$, and IL-18 in the nucleus pulposus (NP) of healthy donors and IDD patients were measured by western blotting analysis. (B) Quantification of the protein expression levels in each group. Data represent mean $\pm \mathrm{SD} ;{ }^{* *} p<0.01$.

miR-141. The synthesis of collagen II and aggrecan was increased by miR-141 inhibition (Figure 4A). The expression levels of acan, sox 9 , and col2 in the miR-mimic group were significantly lower than those in the mimic-NC group. The miR-inhibitor + acid group had higher expression levels of these genes than the inhibitor-NC + acid group (Figure 4B). Also, the expression of ADAMT4, MMP3, and MMP13 was increased in the miRmimic group and decreased in the miR-inhibitor group compared with the respective NC groups (Figure 4C). Alcian blue staining indicated a lower GAG content in the miR-mimic group than that in the mimic-NC group. An acidic environment inhibited GAG deposition; the GAG content in the miR-inhibitor group was higher than that in the inhibitor-NC group (Figure 4D).

\section{miR-141 Modulated Pyroptosis in NPCs by Targeting ROS and TXNIP/NLRP3 Pathway}

We investigated the mechanism underlying miR-141-induced pyroptosis. The reactive oxygen species (ROS) level in the degeneration group was higher than that in the normal group (Figure 5A). miR-141 significantly increased intracellular ROS generation with and without acid compared with the control group. However, the miR-141 inhibitor decreased ROS generation by NPCs in an acidic environment (Figure 5B). NAC decreased ROS generation by NPCs in the mimic-NC and miR-mimic groups (Figure 5C). Furthermore, the pyroptosis rate and caspase- 1 activity in the miR-mimic group were markedly higher than those in the mimic-NC group. NAC downregulated the pyroptosis rate in the mimic-NC + NAC and miR-mimic + NAC groups compared with the mimicNC and miR-mimic groups, respectively (Figures 5D,E). The miR-mimic group also showed increased protein levels of TXNIP, NLRP3, cleaved caspase-1, and NT-GSDMD compared with the mimic-NC group. After the addition of NAC, the mimic-NC and miR-mimic groups showed decreased protein levels of TXNIP, NLRP3, cleaved caspase-1, and NTGSDMD (Figure 5F).

\section{An miR-141 Inhibitor Prevented the Degeneration of IVDs}

The ability of miR-141 to prevent IVD degeneration was verified in vivo. We first assayed pyroptosis in the rat disk degeneration model. The protein levels of cleaved caspase-1, NT-GSDMD, IL-1 $\beta$, and IL-18 in the degeneration group were significantly higher than those in the normal group (Figure 6A). Also, the degeneration group had a higher ROS level than the normal group (Figure 6B). H\&E staining showed that the lamellar sheets of the AF in the normal group were regular and that the NP was well organized with cells and ECM. No regular structure of the AF and NP was observed in the NC and miR-141 mimic groups. The NP in the miR-141 inhibitor group was more regular and well organized than that in the NC group. Safranin O staining, indicating GAG deposition, was stronger in the control and miR-141 inhibitor groups than those in the NC and miR141 mimic groups. Immunohistochemical staining showed the presence of collagen II in the NP of only the normal and miR-141 inhibitor groups. Few areas of the NP were immunepositive for collagen II in the NC and miR-141 mimic groups (Figure 6C). The GAG contents at weeks 0 and 16 in the normal group were significantly higher than those in the other groups. The GAG contents in the NC, miR-141 mimic, and miR-141 inhibitor groups were similar at week 0 . However, the miR-141 inhibitor group showed a higher GAG content than the NC and miR-141 mimic groups at week 16 (Figure 6D). Histological scoring was performed to evaluate the degeneration of IVDs. The score of the control was $5.4 \pm 0.55$, significantly lower than those in the other groups. The miR-141 mimic group had the highest score (13.4 \pm 0.55$)$. The histological score of the miR-141 
A

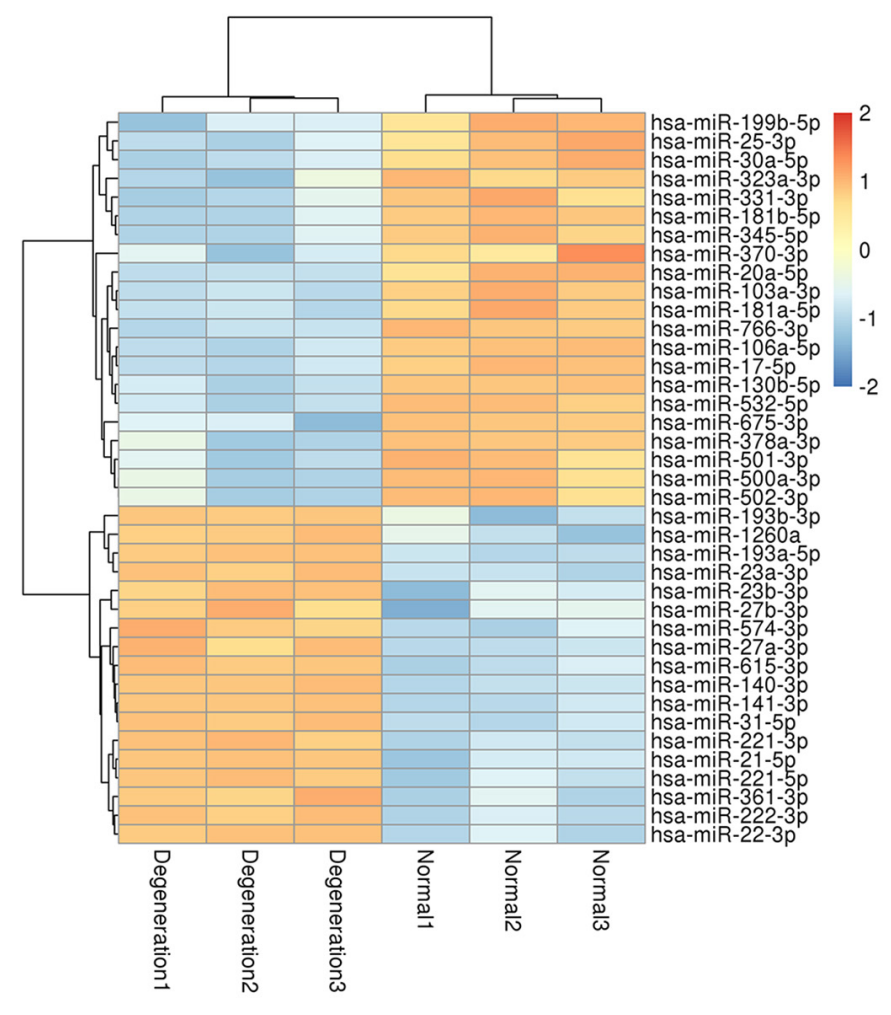

B

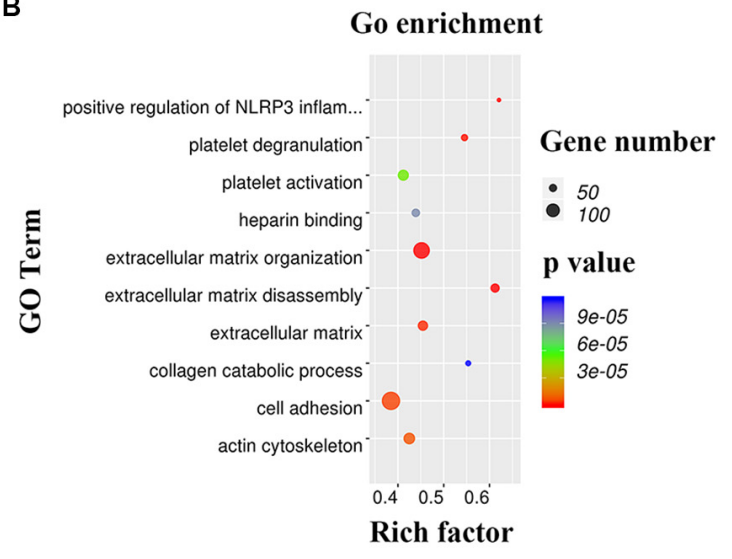

C

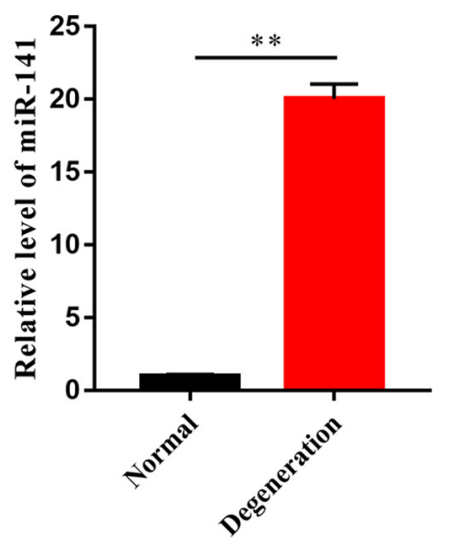

FIGURE 2 | miR-141 was highly expressed in degenerated nucleus pulposus (NP). (A) The heatmap showed differentially expressed MicroRNAs (miRNAs) between normal and degenerated NP. (B) Gene ontology (GO) terms with significant $p$ values for biological processes, molecular function, cellular component. (C) The expression level of miR-141 in normal and degenerated NP was verified by polymerase chain reaction (PCR) and normalized to U6. Data represent mean \pm SEM; $* * p<0.01$.

inhibitor group $(7.6 \pm 1.14)$ was lower than that of the NC group $(12.8 \pm 0.84$; Figure $6 \mathrm{E})$.

\section{DISCUSSION}

Nucleus pulposus degeneration, which is caused by disruption of ECM anabolism and catabolism of NPCs, is the trigger for IVD degeneration (Fontana et al., 2015). Pyroptosis is a form of programed cell death and is accompanied by an inflammatory response (Hu et al., 2018). It plays an important role in regulating cell viability and mediating ECM deposition (Zhaolin et al., 2019). However, the role of pyroptosis in IVD degeneration is unclear. We hypothesize that pyroptosis occurs during IVD degeneration and modulates the viability of, and ECM synthesis by, NPCs. miRNAs play critical roles in cell proliferation, function, and pyroptosis. We investigated the relationship between NP degeneration, pyroptosis, and miRNA. We also investigated the mechanism underlying the miRNAmediated regulation of pyroptosis in NPCs.
In some cases, the pyroptosis pathway can trigger apoptosis. The relationship between apoptosis and pyroptosis in NPCs is unclear (Green, 2019). However, pyroptosis and apoptosis co-exist in cardiomyocytes (Fisch et al., 2019; Li et al., 2019). Therefore, we hypothesize that within the same patient, a portion of NPCs undergo apoptosis and the remainder experience pyroptosis. The proportions of NPCs that undergo pyroptosis may depend on the inducing factor and the phase of degeneration, and a molecular switch may control the initiation of pyroptosis and apoptosis (Lee et al., 2018; Fritsch et al., 2019). Pyroptosis is involved in the degeneration of diverse tissues. Age-related macular degeneration is regulated by the activation of NLRP3 inflammasome signaling (Sun et al., 2020). Pyroptosis of chondrocytes is also involved in cartilage degeneration (Hu et al., 2020). However, no study has assessed the relationship between pyroptosis and IVD degeneration. We evaluated pyroptosis in degenerative NP and found that the levels of markers of pyroptosis (such as cleaved caspase-1, NT-GSDMD, IL-1 $\beta$, and IL-18) were significantly increased in degenerative NP compared to normal NP. Therefore, pyroptosis is involved 
A

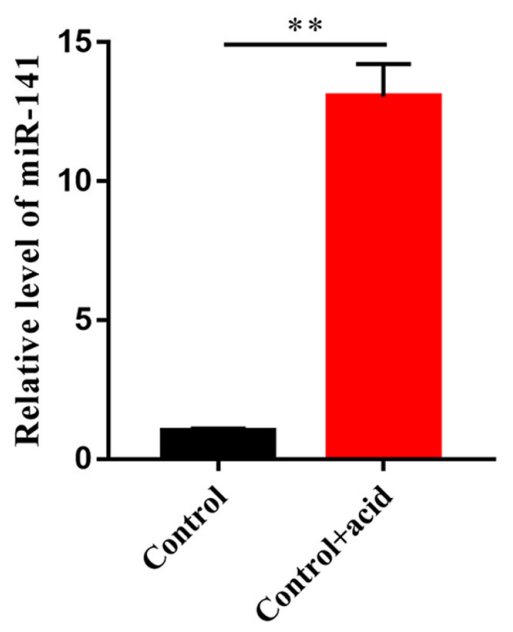

C

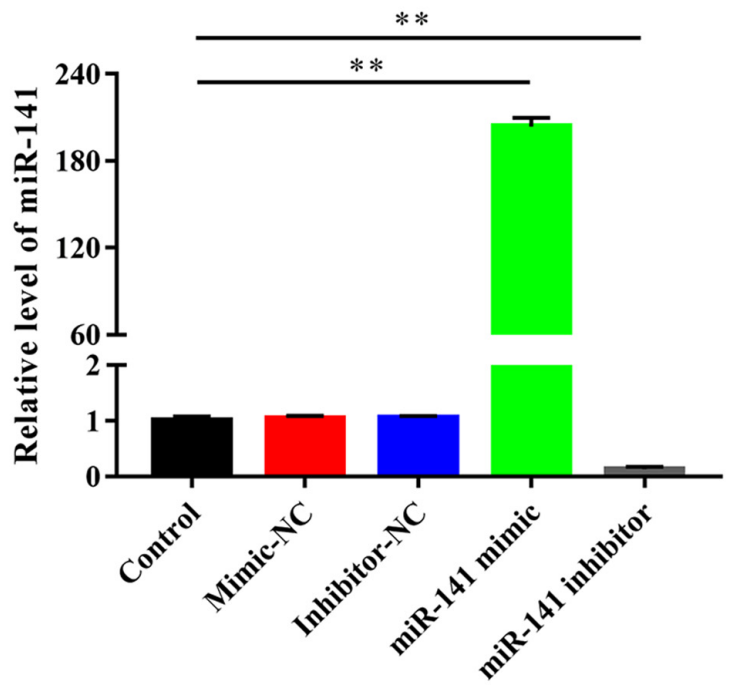

B

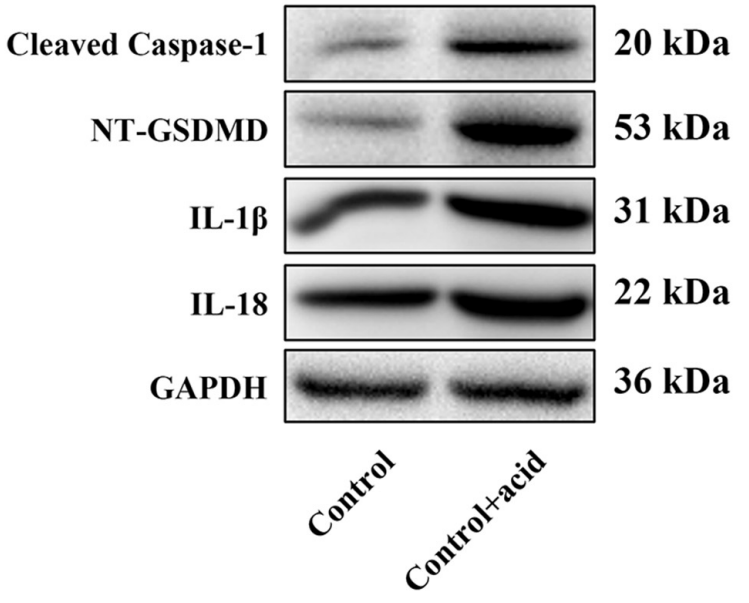

D
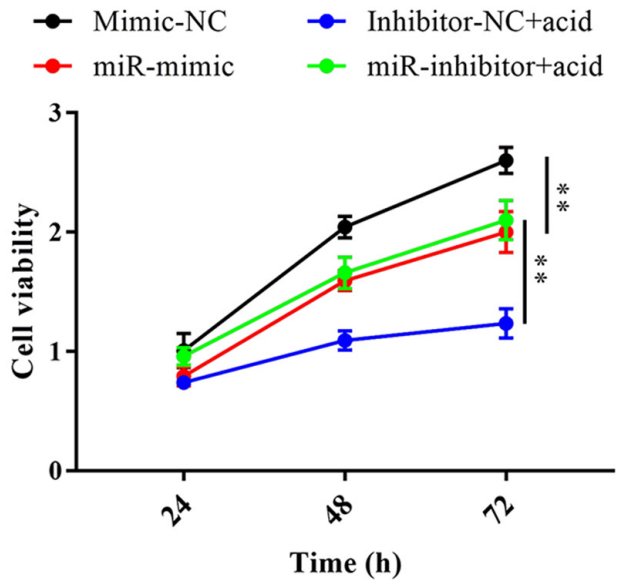

$\mathbf{F}$

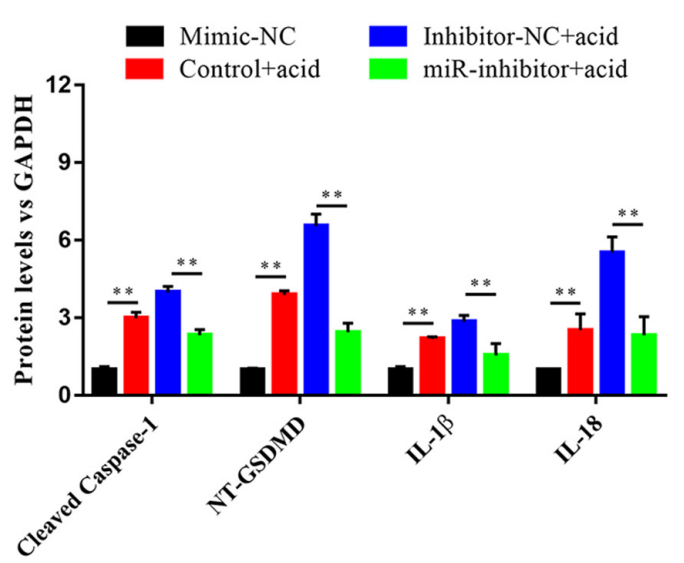

FIGURE 3 | miR-141 induced pyroptosis in nucleus pulposus cells (NPCs). (A) The expression level of miR-141 in the control and control + acid groups. (B) Protein expression levels of Cleaved caspase-1, NT-GSDMD, IL-1 $\beta$, and IL-18 in the control and control + acid groups were measured. (C) NPCs were transfected with miR-NC, miR-141 mimic, and miR-141 inhibitor. The expression levels of miR-141 was measured by polymerase chain reaction (PCR) and normalized to U6. (D) Cell viability after transfection was measured by Cell Counting Kit-8 (CCK8). (E) Protein expression levels of Cleaved caspase-1, NT-GSDMD, IL-1 $\beta$, and IL-18 in each group on day 3 were measured. (F) Quantification of the protein expression levels in each group. Data represent mean $\pm \mathrm{SEM}$; ${ }^{* *} p<0.01$. 


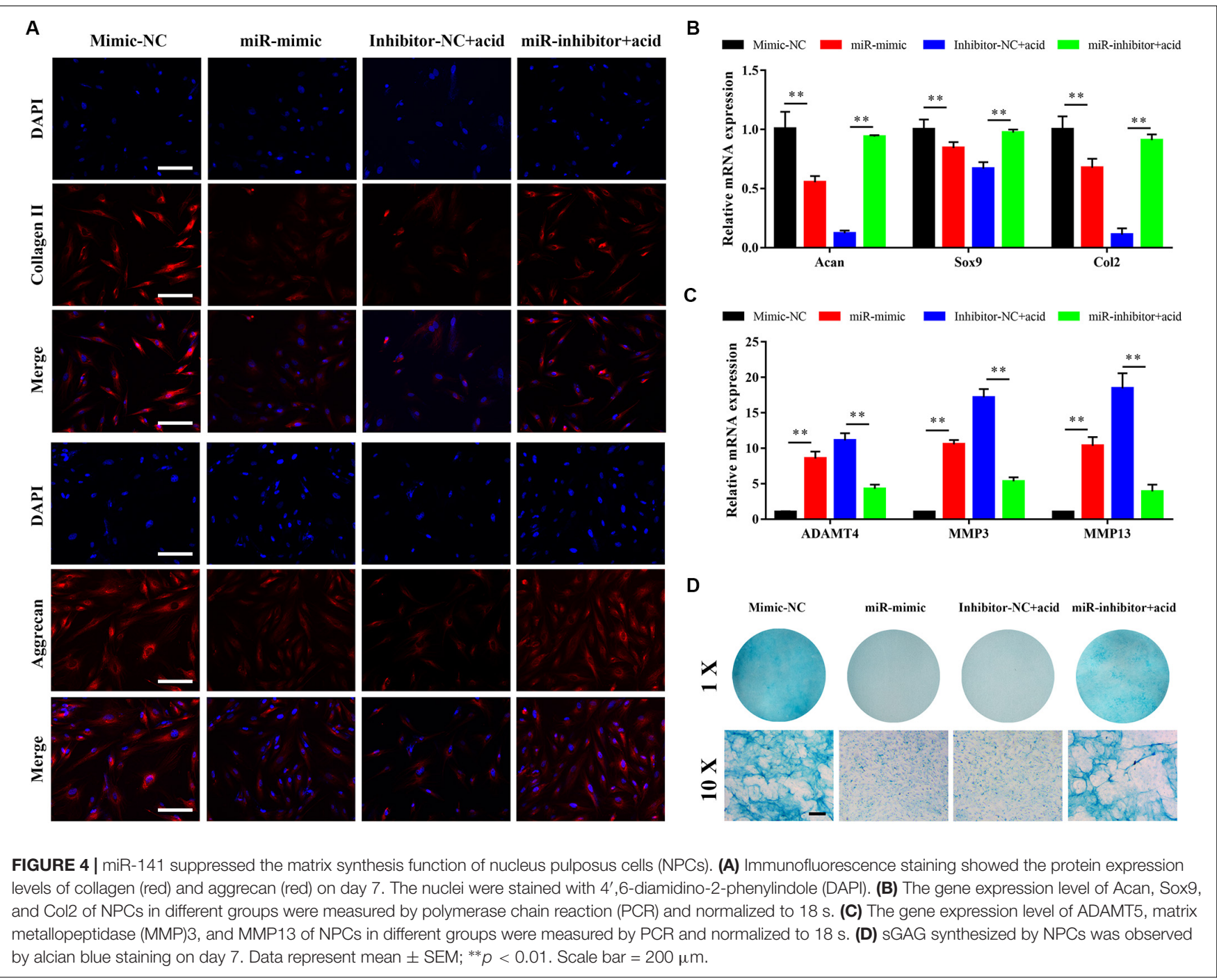

in the degeneration of IVDs. A low $\mathrm{pH}$ increased the levels of cleaved caspase-1, NT-GSDMD, IL-1 $\beta$, and IL-18. Therefore, the acidic microenvironment of degenerative IVD may trigger pyroptosis of NPCs.

We next identified differentially expressed miRNAs between normal and degenerative NP by microarray analysis. The results are consistent with previous studies. For example, miR-222 and miR-21 are reportedly upregulated in degenerative NPCs; this is in agreement with our findings (Wang W.J. et al., 2018; Zhang et al., 2019b). Wang W. et al. (2018) reported that miR-199 is downregulated in degenerated NPCs, also in agreement with our results. The expression of miR-17 in degenerative NPCs was low, which was linked to a higher IVD degeneration grade during inhibition (Song J. et al., 2018). However, the expression levels of some miRNAs reported to be involved in regulating IVD were not significantly different, such as miRNA-124 and miRNA-143 (Wang X.Q. et al., 2019; Yang et al., 2019). This discrepancy may be a result of the source of samples and the systemic error of microarray analysis. miR-141 was significantly upregulated in the degenerative group compared with the normal group by microarray and PCR. miR-141 reportedly regulates apoptosis of NPCs (Ji et al., 2018). Apoptosis of different types of cancer cells mediated by miR-141 has also been reported (Wang N. et al., 2018; Zhang et al., 2019a). The levels of pyroptosis markers were upregulated by an miR-141 mimic and downregulated by an miR-141 inhibitor; by contrast, cell viability was downregulated by an miR-141 mimic and upregulated by an miR-141 inhibitor. These results implicate miR-141 in pyroptosis of NPCs.

We further investigated the mechanism by which miR-141 regulates pyroptosis. The acidic microenvironment in degenerative IVD induces excessive ROS generation (Gonzalez et al., 2020). Indeed, the ROS levels were higher in degenerated NPs compared to normal NPs from human and rat. Also, miRNA-141 increased ROS generation in NPCs and its effect was synergistic with that of acidity. ROS activates TXNIP/NLRPS signaling, which plays an important role in pyroptosis (Chavarria-Smith and Vance, 2015; Gu et al., 2019). In this study, miRNA-141 upregulated the expression of TXNIP and NLRP3 in a ROS-dependent manner. Therefore, miRNA-141 

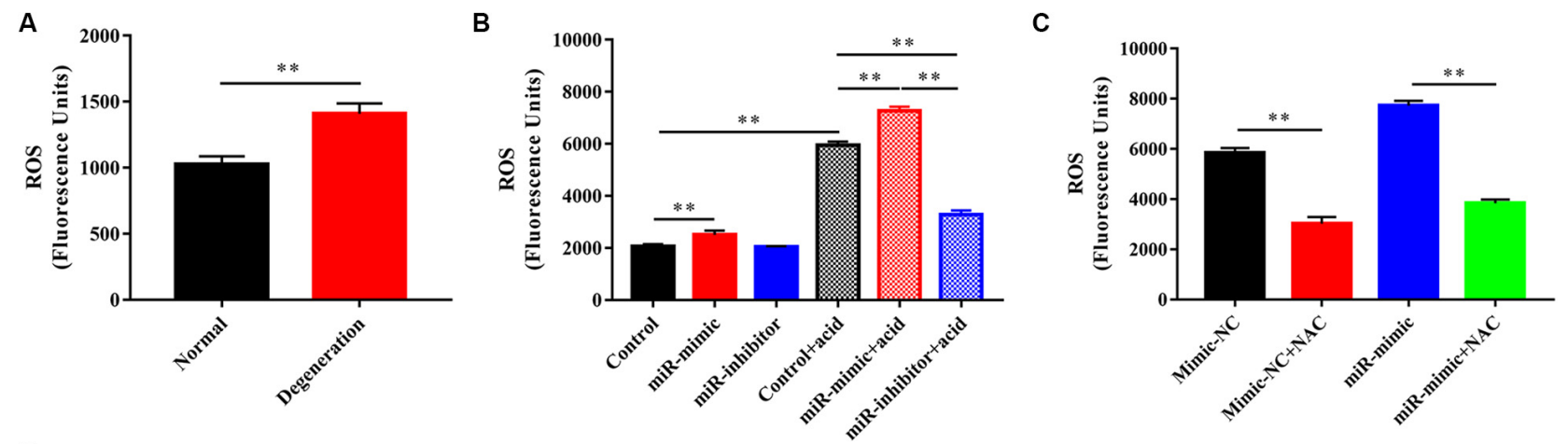

D
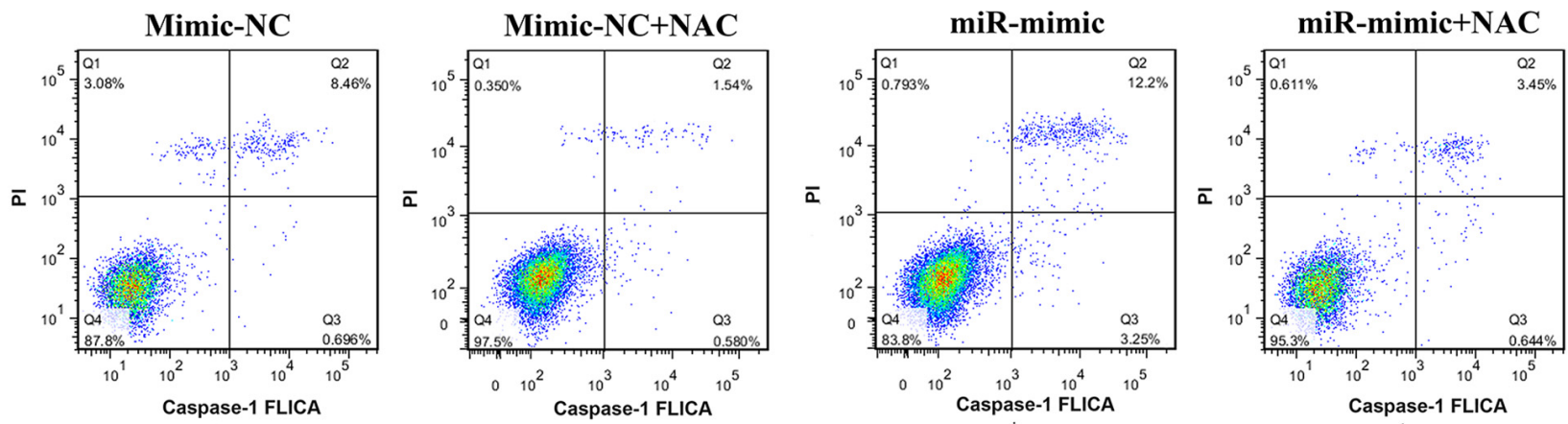

E

$\mathbf{F}$
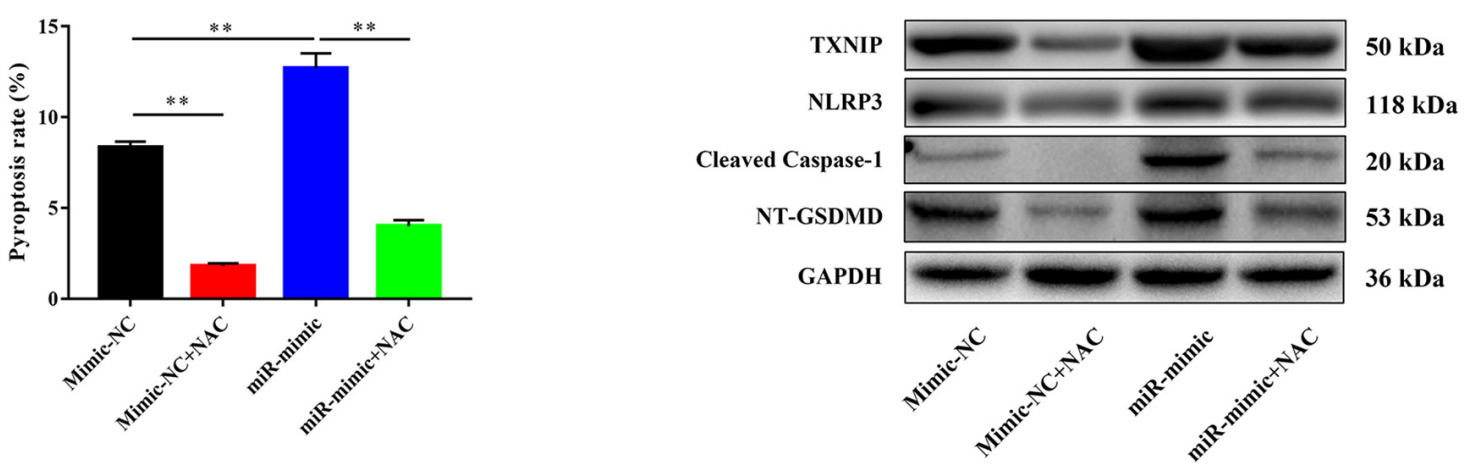

FIGURE 5 | miR-141 modulated pyroptosis in nucleus pulposus cells (NPCs) by targeting reactive oxygen species (ROS) and TXNIP/NLRP3 pathway. (A) The level of ROS in normal and degenerated human nucleus pulposus (NPs). (B) Measurement of intracellular ROS generation in each group using a DCFH-DA probe by fluorometry. (C) N-acetyl cysteine (NAC) was used to decrease the ROS generation, and intracellular ROS generation in each group after using NAC was measured. (D) Pyroptosis in each group was assessed using FLICA/PI staining and flow cytometric analysis. (E) Quantification of pyroptosis rate in each group. (F) Protein expression levels of Cleaved caspase-1, NT-GSDMD, IL-1 1 , and IL-18 in each group after using NAC were measured. Data represent mean \pm SEM; ** $p<0.01$.

induced pyroptosis in NPCs via the ROS/TXNIP/NLRPS axis. However, the mechanism by which miRNA-141 induced ROS generation warrants further investigation.

Extracellular matrix synthesis by NPCs was also modulated by miRNA-141. Acan, Sox9, Col2, MMP3, MMP13, and ADAMT4 reflect the balance between ECM catabolism and anabolism by NPCs (Zhou et al., 2015). The expression levels of Acan, Sox9, and Col2 were downregulated by the miRNA-141 mimic and upregulated by the miRNA-141 inhibitor. By contrast, the expression levels of $M M P 3, M M P 13$, and ADAMT4 were upregulated by the miRNA-141 mimic and downregulated by the miRNA-141 inhibitor. The protein levels of collagen II and aggrecan, and GAG synthesis by NPCs indicated that miRNA-141 inhibited ECM anabolism and aggravated its catabolism by NPCs (Wang X. et al., 2018; Wang Y. et al., 2019). The NF-кB signaling pathway is a key regulator of ECM deposition; its activation upregulates matrix metallopeptidase (MMP) 3 and MMP13 expression and downregulates that of collagen II in chondrocytes (Hu et al., 2020). miRNA-141 increased the generation of ROS, which activate the NF- $\mathrm{KB}$ signaling pathway, and increased the expression of MMPs, inducing ECM degradation (Wang X. et al., 2018; Wang Y. et al., 2019). miRNA-141 itself is 
A

Cleaved Caspase-1

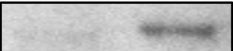

NT-GSDMD

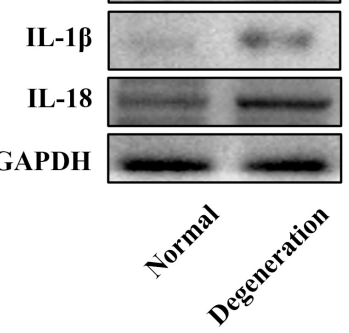

C
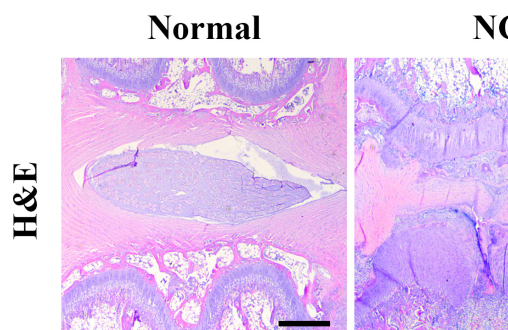

$20 \mathrm{kDa}$

53 kDa

$31 \mathrm{kDa}$

22 kDa

$36 \mathrm{kDa}$
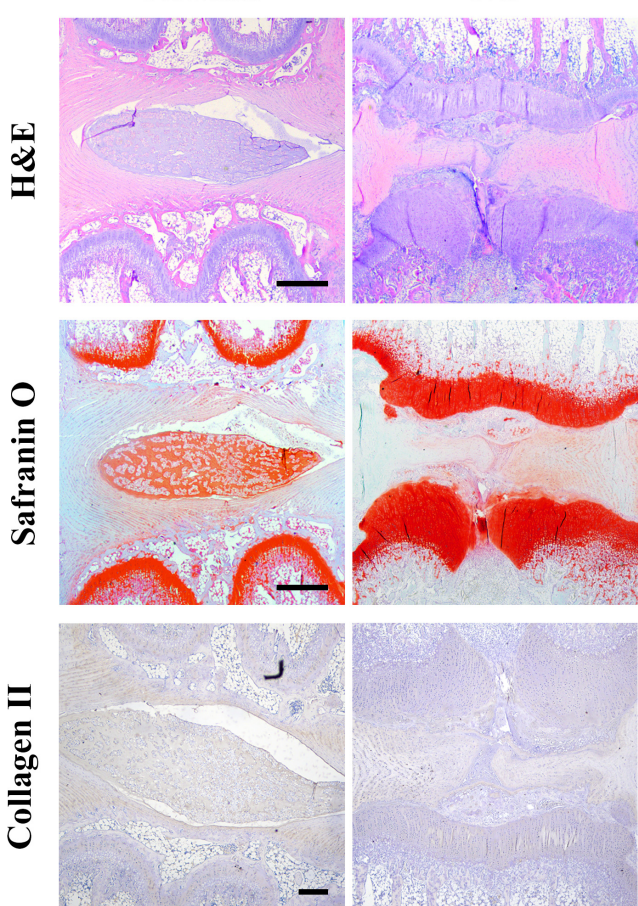

D

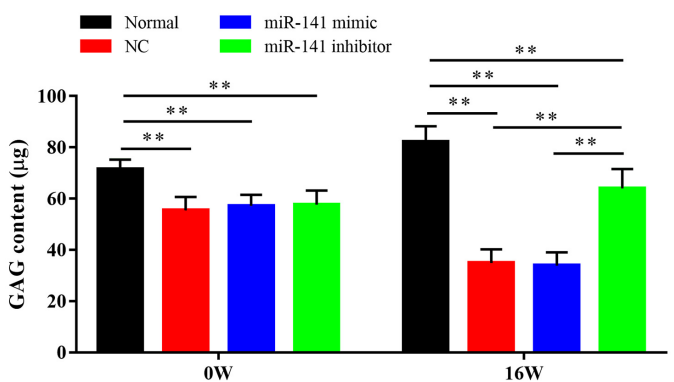

B

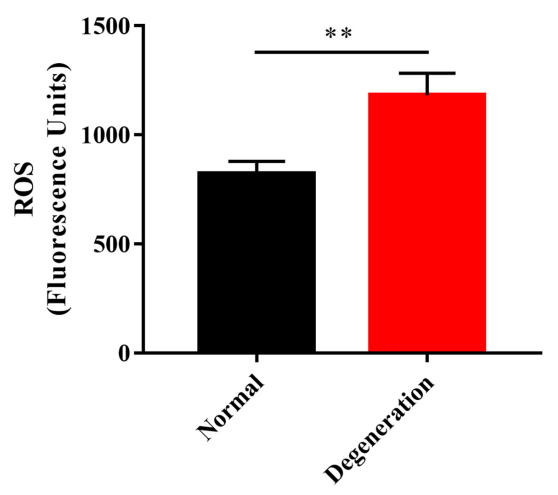

miR-141 mimic miR-141 inhibitor
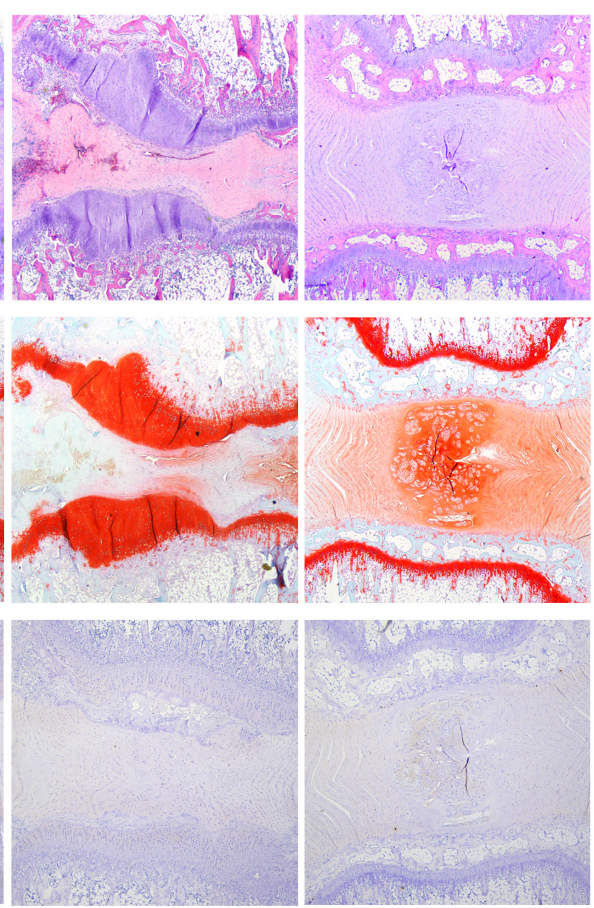

E

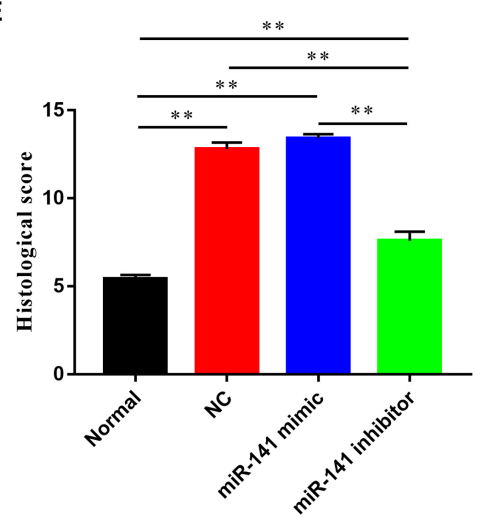

FIGURE 6 | miR-131 inhibitor prevented the degeneration of intervertebral disks (IVDs). (A) The pyroptosis in the degenerated nucleus pulposus (NPs) of rat model was measured by western blotting analysis. (B) The level of ROS in normal and degenerated rat NPs. (C) Representative haematoxylin and eosin (H\&E) and Safranin O staining, and immunohistochemical detection of collagen II of disks from different groups were observed. All samples were harvested at 16 weeks after injection. (D) The content of SGAG in each group at 0 and 16 weeks after injection were quantified by Blyscan assay. (E) Histological grade of each group was evaluated at 16 weeks after injection. Data represent mean \pm SEM; ${ }^{* *} p<0.01$. Scale bar $=500 \mu \mathrm{m}$. 
a positive regulator of NF- $\mathrm{B}$ signaling pathway (Ji et al., 2018). Therefore, NF- $\kappa \mathrm{B}$ signaling regulated by miRNA-141 may be responsible for ECM catabolism by NPCs.

In vivo, pyroptosis occurred in the rat disk degeneration model. The degenerative IVD had an abnormal NP structure and decreased GAG and collagen II contents. The miRNA-141 mimic aggravated IVD degeneration, which also demonstrated the negative regulator of miRNA-141 in NPCs. The miRNA141 inhibitor group had NP of a more regular shape and higher collagen II and GAG contents. However, the differences between normal and miRNA-141 inhibitor-treated IVD indicated that miRNA-141 inhibitors cannot regenerate degenerated IVD. IVD degeneration is modulated by nutrition, mechanical loading, and genetics (Urban and Roberts, 2003; Urban et al., 2004; Navone et al., 2018). The miRNA-141 inhibitor suppressed pyroptosis, upregulated ECM synthesis by NPCs, and prevented IVD degeneration. Therefore, inhibition of pyroptosis may contribute to IVD degeneration treatment, but other modalities are needed.

\section{CONCLUSION}

miRNA-141 induced pyroptosis and ECM catabolism of NPCs. miRNA-141 increased ROS generation in NPCs and activated TXNIP/NLRP3 signaling, leading to pyroptosis. Also, miR-141 inhibitor prevented IVD degeneration in vivo. Our work will attract attention to pyroptosis in IVD and facilitate the discovery of more efficacious treatments for IVD degeneration.

\section{REFERENCES}

Barakat, A. H., Elwell, V. A., and Lam, K. S. (2019). Stem cell therapy in discogenic back pain. J. Spine Surg. 5, 561-583. doi: 10.21037/jss.2019.09.22

Bartel, D. P. (2009). MicroRNAs: target recognition and regulatory functions. Cell 136, 215-233. doi: 10.1016/j.cell.2009.01.002

Bowles, R. D., and Setton, L. A. (2017). Biomaterials for intervertebral disc regeneration and repair. Biomaterials 129, 54-67. doi: 10.1016/j.biomaterials. 2017.03.013

Chavarria-Smith, J., and Vance, R. E. (2015). The NLRP1 inflammasomes. Immunol. Rev. 265, 22-34. doi: 10.1111/imr.12283

Eser, B., Eser, O., Aslan, E., and Dolgun, H. (2017). The effects of polymorphisms of death pathway genes and mitochondrial pathway genes in intervertebral disc degeneration. Turk. Neurosurg. 27, 809-815.

Fisch, D., Bando, H., Clough, B., Hornung, V., Yamamoto, M., Shenoy, A. R., et al. (2019). Human GBP1 is a microbe-specific gatekeeper of macrophage apoptosis and pyroptosis. EMBO J. 38:e100926.

Fontana, G., See, E., and Pandit, A. (2015). Current trends in biologics delivery to restore intervertebral disc anabolism. Adv. Drug Deliv. Rev. 84, 146-158. doi: 10.1016/j.addr.2014.08.008

Fritsch, M., Gunther, S. D., Schwarzer, R., Albert, M. C., Schorn, F., Werthenbach, J. P., et al. (2019). Caspase-8 is the molecular switch for apoptosis, necroptosis and pyroptosis. Nature 575, 683-687. doi: 10.1038/s41586-019-1770-6

$\mathrm{Fu}$, J., Yu, W., and Jiang, D. (2018). Acidic $\mathrm{pH}$ promotes nucleus pulposus cell senescence through activating the p38 MAPK pathway. Biosci. Rep. 38:BSR20181451.

Gonzalez, D., Alamos, P., Rivero, M., Orellana, O., Norambuena, J., Chavez, R., et al. (2020). Deciphering the role of multiple thioredoxin fold proteins of leptospirillum sp. in oxidative stress tolerance. Int. J. Mol. Sci. 21:1880. doi: $10.3390 /$ ijms 21051880

\section{DATA AVAILABILITY STATEMENT}

The original contributions presented in the study are included in the article/supplementary material, further inquiries can be directed to the corresponding authors.

\section{ETHICS STATEMENT}

The animal study was reviewed and approved by Ethics Committee of The Second Affiliated Hospital of Zhejiang University School of Medicine. Written informed consent was obtained from the individual(s) for the publication of any potentially identifiable images or data included in this article.

\section{AUTHOR CONTRIBUTIONS}

QX, WC, and NZ designed the research. QX performed the research, analyzed the data, and wrote the manuscript. HX performed the research and analyzed the data. JW performed the research. All authors contributed to the article and approved the submitted version.

\section{FUNDING}

This work was supported by the National Natural Science Foundation of China (81603126 and 81972514) to NZ.

Green, D. R. (2019). The coming decade of cell death research: five riddles. Cell 177, 1094-1107. doi: 10.1016/j.cell.2019.04.024

Gu, C., Draga, D., Zhou, C., Su, T., Zou, C., Gu, Q., et al. (2019). miR-590-3p inhibits pyroptosis in diabetic retinopathy by targeting NLRP1 and inactivating the NOX4 signaling pathway. Invest. Ophthalmol. Vis. Sci. 60, 4215-4223.

Han, B., Zhu, K., Li, F. C., Xiao, Y. X., Feng, J., Shi, Z. L., et al. (2008). A simple disc degeneration model induced by percutaneous needle puncture in the rat tail. Spine 33, 1925-1934. doi: 10.1097/brs.0b013e31817c64a9

Hartman, R., Patil, P., Tisherman, R., St Croix, C., Niedernhofer, L. J., Robbins, P. D., et al. (2018). Age-dependent changes in intervertebral disc cell mitochondria and bioenergetics. Eur. Cell Mater. 36, 171-183. doi: 10.22203/ ecm.v036a13

Hu, J., Zhou, J., Wu, J., Chen, Q., Du, W., Fu, F., et al. (2020). Loganin ameliorates cartilage degeneration and osteoarthritis development in an osteoarthritis mouse model through inhibition of NF-kappaB activity and pyroptosis in chondrocytes. J. Ethnopharmacol. 247:112261. doi: 10.1016/j.jep.2019.112261

Hu, Q., Zhang, T., Yi, L., Zhou, X., and Mi, M. (2018). Dihydromyricetin inhibits NLRP3 inflammasome-dependent pyroptosis by activating the Nrf2 signaling pathway in vascular endothelial cells. Biofactors 44, 123-136. doi: 10.1002/biof. 1395

Ji, M. L., Jiang, H., Zhang, X. J., Shi, P. L., Li, C., Wu, H., et al. (2018). Preclinical development of a microRNA-based therapy for intervertebral disc degeneration. Nat. Commun. 9:5051.

Jin, L. Z., Lu, J. S., and Gao, J. W. (2018). Silencing SUMO2 promotes protection against degradation and apoptosis of nucleus pulposus cells through p53 signaling pathway in intervertebral disc degeneration. Biosci. Rep. 38:BSR20171523.

Kepler, C. K., Ponnappan, R. K., Tannoury, C. A., Risbud, M. V., and Anderson, D. G. (2013). The molecular basis of intervertebral disc degeneration. Spine J. $13,318-330$. 
Kong, L., Sun, M., Jiang, Z., Li, L., and Lu, B. (2018). MicroRNA-194 inhibits lipopolysaccharide-induced inflammatory response in nucleus pulposus cells of the intervertebral disc by targeting TNF receptor-associated factor 6 (TRAF6). Med. Sci. Monit. 24, 3056-3067. doi: 10.12659/msm.907280

Kong, Q., Liang, C., Jin, Y., Pan, Y., Tong, D., Kong, Q., et al. (2019). The IncRNA MIR4435-2HG is upregulated in hepatocellular carcinoma and promotes cancer cell proliferation by upregulating miRNA-487a. Cell Mol. Biol. Lett. 24:26.

Lee, S., Hirohama, M., Noguchi, M., Nagata, K., and Kawaguchi, A. (2018). Influenza A Virus infection triggers pyroptosis and apoptosis of respiratory epithelial cells through the Type I interferon signaling pathway in a mutually exclusive manner. J. Virol. 92:e00396-18.

Li, N., Zhou, H., Wu, H., Wu, Q., Duan, M., Deng, W., et al. (2019). STING-IRF3 contributes to lipopolysaccharide-induced cardiac dysfunction, inflammation, apoptosis and pyroptosis by activating NLRP3. Redox Biol. 24:101215. doi: 10.1016/j.redox.2019.101215

Li, P., Xu, T., Zhou, X., Liao, L., Pang, G., Luo, W., et al. (2017). Downregulation of miRNA-141 in breast cancer cells is associated with cell migration and invasion: involvement of ANP32E targeting. Cancer Med. 6, 662-672. doi: 10.1002/cam4. 1024

Liu, X., Zhang, Z., Ruan, J., Pan, Y., Magupalli, V. G., Wu, H., et al. (2016). Inflammasome-activated gasdermin $\mathrm{D}$ causes pyroptosis by forming membrane pores. Nature 535, 153-158. doi: 10.1038/nature18629

Liu, W., Zhang, Y., Xia, P., Li, S., Feng, X., Gao, Y., et al. (2016). MicroRNA7 regulates IL-1beta-induced extracellular matrix degeneration by targeting GDF5 in human nucleus pulposus cells. Biomed. Pharmacother. 83, 1414-1421. doi: 10.1016/j.biopha.2016.08.062

Manchikanti, L., Singh, V., Datta, S., Cohen, S. P., Hirsch, J. A., and American Society of Interventional Pain Physicians (2009). Comprehensive review of epidemiology, scope, and impact of spinal pain. Pain Phys. 12, E35-E70.

Masuda, K., Aota, Y., Muehleman, C., Imai, Y., Okuma, M., Thonar, E. J., et al. (2005). A novel rabbit model of mild, reproducible disc degeneration by an anulus needle puncture: correlation between the degree of disc injury and radiological and histological appearances of disc degeneration. Spine 30, 5-14. doi: 10.1097/01.brs.0000148152.04401.20

Mathew, J., Singh, S. B., Garis, S., and Diwan, A. D. (2013). Backing up the stories: the psychological and social costs of chronic low-back pain. Int. J. Spine Surg. 7, e29-e38. doi: 10.1016/j.ijsp.2013.02.001

Navone, S. E., Peroglio, M., Guarnaccia, L., Beretta, M., Grad, S., Paroni, M., et al. (2018). Mechanical loading of intervertebral disc modulates microglia proliferation, activation, and chemotaxis. Osteoarthr. Cartil. 26, 978-987. doi: 10.1016/j.joca.2018.04.013

Qin, Q., Cui, L., Zhou, Z., Zhang, Z., Wang, Y., and Zhou, C. (2019). Inhibition of microRNA-141-3p reduces hypoxia-induced apoptosis in H9c2 rat cardiomyocytes by activating the RP105-dependent PI3K/AKT signaling pathway. Med. Sci. Monit. 25, 7016-7025. doi: 10.12659/msm.916361

Ruhl, S., Shkarina, K., Demarco, B., Heilig, R., Santos, J. C., and Broz, P. (2018). ESCRT-dependent membrane repair negatively regulates pyroptosis downstream of GSDMD activation. Science 362, 956-960. doi: 10.1126/science. aar7607

Song, J., Wang, H. L., Song, K. H., Ding, Z. W., Wang, H. L., Ma, X. S., et al. (2018). CircularRNA_104670 plays a critical role in intervertebral disc degeneration by functioning as a ceRNA. Exp. Mol. Med. 50:94.

Song, Y., Li, S., Geng, W., Luo, R., Liu, W., Tu, J., et al. (2018). Sirtuin 3-dependent mitochondrial redox homeostasis protects against AGEs-induced intervertebral disc degeneration. Redox Biol. 19, 339-353. doi: 10.1016/j.redox.2018. 09.006

Sun, H. J., Jin, X. M., Xu, J., and Xiao, Q. (2020). Baicalin alleviates age-related macular degeneration via miR-223/NLRP3-regulated pyroptosis. Pharmacology 105, 28-38. doi: 10.1159/000502614

Sun, Z., Jian, Y., Fu, H., and Li, B. (2018). MiR-532 downregulation of the Wnt/beta-catenin signaling via targeting Bcl-9 and induced human intervertebral disc nucleus pulposus cells apoptosis. J. Pharmacol. Sci. 138, 263-270. doi: 10.1016/j.jphs.2018.10.007

Urban, J. P., and Roberts, S. (2003). Degeneration of the intervertebral disc. Arthrit. Res. Ther. 5, 120-130.
Urban, J. P., Smith, S., and Fairbank, J. C. (2004). Nutrition of the intervertebral disc. Spine 29, 2700-2709.

Wang, N., Li, P., Liu, W., Wang, N., Lu, Z., Feng, J., et al. (2018). miR-141$3 p$ suppresses proliferation and promotes apoptosis by targeting GLI2 in osteosarcoma cells. Oncol. Rep. 39, 747-754.

Wang, W., Guo, Z., Yang, S., Wang, H., and Ding, W. (2018). Upregulation of miR-199 attenuates TNF-alpha-induced human nucleus pulposus cell apoptosis by downregulating MAP3K5. Biochem. Biophys. Res. Commun. 505, 917-924. doi: 10.1016/j.bbrc.2018.09.194

Wang, W. J., Yang, W., Ouyang, Z. H., Xue, J. B., Li, X. L., Zhang, J., et al. (2018). MiR-21 promotes ECM degradation through inhibiting autophagy via the PTEN/akt/mTOR signaling pathway in human degenerated NP cells. Biomed. Pharmacother. 99, 725-734. doi: 10.1016/j.biopha.2018.01.154

Wang, X., Zou, M., Li, J., Wang, B., Zhang, Q., Liu, F., et al. (2018). LncRNA H19 targets miR-22 to modulate $\mathrm{H} 2 \mathrm{O} 2$-induced deregulation in nucleus pulposus cell senescence, proliferation, and ECM synthesis through Wnt signaling. J. Cell Biochem. 119, 4990-5002. doi: 10.1002/jcb.26738

Wang, X. Q., Tu, W. Z., Guo, J. B., Song, G., Zhang, J., Chen, C. C., et al. (2019). A bioinformatic analysis of MicroRNAs' role in human intervertebral disc degeneration. Pain Med. 20, 2459-2471. doi: 10.1093/pm/pnz015

Wang, Y., Wang, L., Wen, X., Hao, D., Zhang, N., He, G., et al. (2019). NF-kappaB signaling in skin aging. Mech. Ageing Dev. 184:111160.

Xue, Y., Enosi Tuipulotu, D., Tan, W. H., Kay, C., and Man, S. M. (2019). Emerging activators and regulators of inflammasomes and pyroptosis. Trends Immunol. 40, 1035-1052. doi: 10.1016/j.it.2019.09.005

Yang, Q., Guo, X. P., Cheng, Y. L., and Wang, Y. (2019). MicroRNA-143-5p targeting eEF2 gene mediates intervertebral disc degeneration through the AMPK signaling pathway. Arthrit. Res. Ther. 21:97.

Yu, Z. W., Zhang, J., Li, X., Wang, Y., Fu, Y. H., and Gao, X. Y. (2020). A new research hot spot: the role of NLRP3 inflammasome activation, a key step in pyroptosis, in diabetes and diabetic complications. Life Sci. 240:117138. doi 10.1016/j.lfs.2019.117138

Zeng, R., Luo, D. X., Li, H. P., Zhang, Q. S., Lei, S. S., and Chen, J. H. (2019). MicroRNA-135b alleviates MPP(+)-mediated Parkinson's disease in in vitro model through suppressing FoxO1-induced NLRP3 inflammasome and pyroptosis. J. Clin. Neurosci. 65, 125-133. doi: 10.1016/j.jocn.2019.04.004

Zhang, Y., Yan, J., and Pan, X. (2019a). miR-141-3p affects apoptosis and migration of endometrial stromal cells by targeting KLF-12. Pflugers Arch. 471, 1055-1063. doi: 10.1007/s00424-019-02283-2

Zhang, Y., Yang, J., Zhou, X., Wang, N., Li, Z., Zhou, Y., et al. (2019b). Knockdown of miR-222 inhibits inflammation and the apoptosis of LPS-stimulated human intervertebral disc nucleus pulposus cells. Int. J. Mol. Med. 44, 1357-1365.

Zhao, G., Wang, B., Liu, Y., Zhang, J. G., Deng, S. C., Qin, Q., et al. (2013). miRNA-141, downregulated in pancreatic cancer, inhibits cell proliferation and invasion by directly targeting MAP4K4. Mol. Cancer Ther. 12, 2569-2580. doi: 10.1158/1535-7163.mct-13-0296

Zhaolin, Z., Guohua, L., Shiyuan, W., and Zuo, W. (2019). Role of pyroptosis in cardiovascular disease. Cell Prolif. 52:e12563. doi: 10.1111/cpr.12563

Zheng, X., Zhong, T., Ma, Y., Wan, X., Qin, A., Yao, B., et al. (2020). Bnip3 mediates doxorubicin-induced cardiomyocyte pyroptosis via caspase-3/GSDME. Life Sci. 242:117186. doi: 10.1016/j.lfs.2019.117186

Zhou, X., Tao, Y., Liang, C., Zhang, Y., Li, H., and Chen, Q. (2015). BMP3 alone and together with TGF-beta promote the differentiation of human mesenchymal stem cells into a nucleus pulposus-like phenotype. Int. J. Mol. Sci. 16, 2034420359. doi: 10.3390/ijms160920344

Conflict of Interest: The authors declare that the research was conducted in the absence of any commercial or financial relationships that could be construed as a potential conflict of interest.

Copyright (c) $2020 \mathrm{Xu}$, Xing, Wu, Chen and Zhang. This is an open-access article distributed under the terms of the Creative Commons Attribution License (CC BY). The use, distribution or reproduction in other forums is permitted, provided the original author(s) and the copyright owner(s) are credited and that the original publication in this journal is cited, in accordance with accepted academic practice. No use, distribution or reproduction is permitted which does not comply with these terms. 